\title{
Complication of transvenous lead extraction: migration of the broken helix of an implantable cardioverter-defibrillator lead to the hepatic vein
}

\author{
Mateusz Ulman' ${ }^{1}$, Maciej Dębski ${ }^{1}$, Krzysztof Boczar ${ }^{1}$, Andrzej Ząbek ${ }^{1}$, \\ Jacek Lelakowski ${ }^{1,2}$, Barbara Małecka ${ }^{1,2}$ \\ 'Department of Electrocardiology, John Paul II Hospital, Krakow, Poland \\ 2Institute of Cardiology, Jagiellonian University Medical College, Krakow, Poland
}

Lead breakage is a potentially life-threatening complication associated with transvenous lead extraction (TLE) and occurs in $4 \%-8 \%$ of surgical procedures [1-3]. Insulation failure, stretching of the inner metal coil, and breakage can occur, usually in places of firm fibrous tissue adherence to the lead, particularly when tissue is calcified. This happens most commonly with strongly ingrown, chronically implanted leads. The risk of lead breakage can be minimised by filling the inside of the lead with a locking stylet. Usually, the distal lead fragment with tip remains fixed to the endocardium with fibrous tissue. The scenario when the distal lead part is broken and wanders with the bloodstream into pulmonary circulation [4], or even into the superolateral vein in the spinal canal causing root compression [5], is extremely rare. We have not identified any reports of the migration of a broken lead fragment to the hepatic vein. We present a case of a 58-year-old man after implantation of a single-chamber implantable cardioverter-defibrillator (ICD) in 2008 for secondary prevention of sudden cardiac death and replacement in 2015, with paroxysmal atrial fibrillation and heart failure with reduced ejection fraction. He was referred for TLE because of inappropriate high-voltage interventions caused by lead damage. The nine-year-old Medtronic Sprint Quattro defibrillator lead was implanted by subclavian vein puncture into the right ventricular outflow tract. In the initial procedure, the lead was filled by the Liberator ${ }^{\circledR}$ Beacon ${ }^{\circledR}$ Tip Locking Stylet guidewire (Cook Medical LLC, Bloomington, IN, USA). Then, using the mechanical system Byrd Dilator Sheaths Telescoping Polypropylene (green, white, and orange; Cook Medical LLC), the lead was released from fibrous tissue adhesions. During TLE the active fixation screw of the tip of ICD lead was broken from lead body and migrated to a branch of the mid hepatic vein (Fig. 1). Removal of the lead fragment was not attempted due to the difficulty in reaching the distal hepatic vein using the available snaring equipment. The patient was implanted a new dual-chamber ICD. Chest X-ray showed the stretched active fixation screw of the tip of the ICD lead in hepatic vessels. He was followed for four days after the procedure in the ward and at six and 12 months at an outpatient clinic. Pacing lead extraction is a technically challenging and risky procedure. Several intravascular techniques for extraction of permanent pacemaker leads have been described, including locking stylets, sheaths, snares, and laser sheaths, each with its own benefits and risks. In older techniques complications of forceful traction include lead fracture or even transection of the superior vena cava. In modern techniques, the lead is filled by the locking stylet; then, using the telescoping sheaths, it is released from fibrous tissue adhesions. In our patient, the extraction was complicated by breakage and migration of the active fixation screw of the tip of the ICD lead, which is not attached to the locking stylet. We wanted to avoid complicated transvenous techniques or surgical removal of the lead fragment. Lead fracture and embolisations of the electrode fragment are known complications of TLE. Our patient did not report any symptoms during follow-up - there were no signs of hepatic embolisation. However, we report this case because of the unusual migration to the branch of the mid hepatic vein. Intravascular techniques for lead extraction including locking stylets and telescoping sheaths significantly reduce the risk of electrode rupture, but helix fracture and embolisation are still possible.

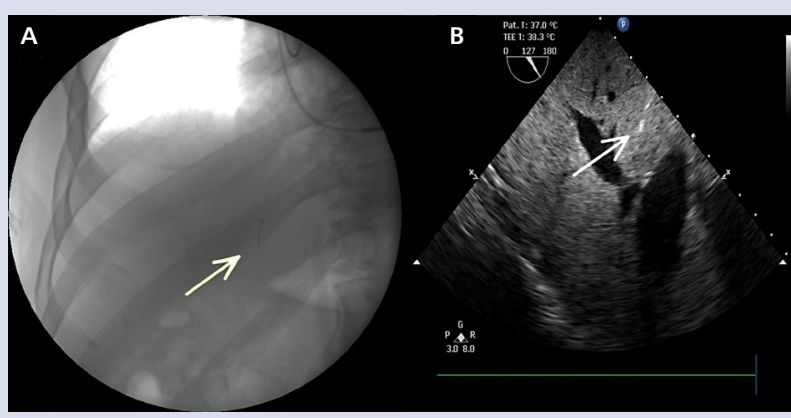

Figure 1. Stretched active fixation helix of the tip of implantable cardioverter-defibrillator lead in the hepatic vessel (arrows). A. Fluoroscopy image; B. Transthoracic echocardiography

\section{References}

1. Fender EA, Killu AM, Cannon BC, et al. Lead extraction outcomes in patients with congenital heart disease. Europace. 2017; 19(3): 441-446, doi: 10.1093/europace/euw049, indexed in Pubmed: 27738059.

2. Gomes S, Cranney G, Bennett M, et al. Long-Term outcomes following transvenous lead extraction. Pacing Clin Electrophysiol. 2016; 39(4): 345-351, doi: 10.1111/pace.12812, indexed in Pubmed: 26768807.

3. Zabek A, Malecka B, Haberka K, et al. The analysis of indications and early results of transvenous lead extraction in patients with a pacemaker, ICD and CRT - single-center experience. Acta Cardiol. 2015; 70(6): 685-692, doi: 10.2143/AC.70.6.3120181, indexed in Pubmed: 26717217.

4. Walters MI, Kaye GC. Pulmonary embolization of a pacing electrode fragment complicating lead extraction. Pacing Clin Electrophysiol. 1999; 22(5): 823-824, indexed in Pubmed: 10353147

5. Yildirir A, Batur MK, Oto A. Embolization of pacing electrode fragment into the superolateral vein in the spinal canal causing root compression. J Cardiovasc Electrophysiol. 2002; 13(3): 290-291, indexed in Pubmed: 11942601.

\section{Address for correspondence:}

Mateusz Ulman, MD, Department of Electrocardiology, John Paul II Hospital, ul. Prądnicka 80, 31-202 Kraków, Poland. tel: +48 126142381 ,

fax: +48 1263323 99, e-mail: mateuszulman@gmail.com

Conflict of interest: none declared

Kardiologia Polska Copyright ( ) Polish Cardiac Society 2018 